\title{
Diagnostics of Eosinophilic Esophagitis: Clinical, Endoscopic, and Histologic Pitfalls
}

\author{
Evan S. Dellon ${ }^{a, b}$ \\ ${ }^{a}$ Center for Esophageal Diseases and Swallowing, and ${ }^{b}$ Center for Gastrointestinal Biology and Disease, Division of \\ Gastroenterology and Hepatology, Department of Medicine, University of North Carolina School of Medicine, \\ Chapel Hill, N.C., USA
}

\section{Key Words}

Eosinophilic esophagitis · Diagnosis · Symptoms .

Endoscopy $\cdot$ Histology $\cdot$ Pathology

\begin{abstract}
Eosinophilic esophagitis (EoE) is currently defined as an immune-mediated chronic esophageal disorder that is diagnosed using both clinical and pathologic information. A series of consensus diagnostic guidelines for EoE have brought a measure of consistency to the field, but in practice the diagnosis of EoE can be challenging. Typical clinical symptoms of EoE, including dysphagia, heartburn, and chest pain, can overlap with gastroesophageal reflux disease, which itself is a common indication for performing endoscopic evaluation. The endoscopic findings of EoE, such as esophageal rings, strictures, linear furrows, and white exudates, are not specific. Esophageal eosinophilia, the histologic hallmark of EoE, is also not pathognomonic and can be seen in a range of conditions. Further complicating the diagnosis of EoE is the newly recognized entity of proton pump inhibitor-responsive esophageal eosinophilia, a condition that must be excluded prior to confirming a diagnosis of EoE. This paper will review the current diagnostic criteria for EoE and discuss multiple clinical, endoscopic, and histologic pitfalls in making the diagnosis of EoE.

(c) 2014 S. Karger AG, Basel
\end{abstract}

() 2014 S. Karger AG, Basel

0257-2753/14/0322-0048\$39.50/0

\section{Introduction}

Eosinophilic esophagitis (EoE) is a chronic inflammatory condition of the esophagus that has become widely recognized as a major cause of upper gastrointestinal morbidity [1]. It is currently defined as an immune/allergy-mediated clinicopathologic condition and is characterized both by symptoms of esophageal dysfunction and a prominent eosinophilic infiltrate in the esophageal $\mathrm{mu}$ cosa [2]. Despite the publication of a series of guidelines over the past 6 years [2-4], diagnosis of EoE is not straightforward and challenges are frequently encountered [5]. The purpose of this paper is to review the current diagnostic guidelines for EoE and present clinical, endoscopic, and histologic pitfalls that are encountered when diagnosing EoE. Clinical pitfalls include inadequately considering the differential diagnosis of esophageal eosinophilia, distinguishing gastroesophageal reflux disease (GERD) and EoE, and appropriately excluding the newly recognized entity of proton pump inhibitorresponsive esophageal eosinophilia (PPI-REE). Endoscopic pitfalls include the reliability of typical findings of EoE and inadequate esophageal biopsy protocols. Histologic pitfalls include variability in diagnostic criteria and in eosinophil count quantification. Techniques and emerging technologies to facilitate the diagnosis of $\mathrm{EoE}$ are addressed in detail in other manuscripts in this issue.

\section{KARGER}

E-Mail karger@karger.com

www.karger.com/ddi
Evan S. Dellon, MD

CB\# 7080, Bioinformatics Building

130 Mason Farm Rd., UNC-CH

Chapel Hill, NC 27599-7080 (USA)

E-Mail edellon@med.unc.edu 


\section{EoE Diagnostic Guidelines}

While the first case report of EoE was published more than 3 decades ago [6] and case series describing the disease as it is currently recognized were published about 20 years ago [7-9], the first guidelines for diagnosis were only published in 2007 [3]. These defined EoE as a clinicopathologic disorder requiring symptoms of esophageal dysfunction and an esophageal biopsy with at least 15 eosinophils per high-power field (eos/HPF), but in the absence of GERD. The guidelines were updated in 2011 by a large consensus committee, and while the symptom and histology requirements were unchanged, there was no longer a requirement to specifically exclude GERD [2]. Instead, the complex interplay between GERD and EoE was acknowledged (see below), and there was a requirement to exclude PPI-REE, a newly recognized cause of esophageal eosinophilia. Most recently, clinical guidelines from the American College of Gastroenterology reinforce the 2011 diagnostic guidelines while emphasizing the importance of excluding secondary causes of esophageal eosinophilia [4].

Despite the publication of these guidelines, practice pattern surveys indicate that at most one third of gastroenterologists and allergists followed the 2007 guidelines $[10,11]$. Similarly, a third of the publications have strictly adhered to the guidelines [12]. These results may be explained, in part, by challenges that are embedded in each of the diagnostic criteria. In practice, it is common to encounter clinical, endoscopic, and histologic pitfalls when attempting the diagnosis of EoE. A major reason for this is that there is no single clinical, endoscopic, or histologic sign that is pathognomonic for EoE, and the entire clinical picture must be considered before a diagnosis can be made.

\section{Clinical Pitfalls in EoE Diagnosis}

The first pitfall encountered in EoE diagnosis is failing to consider the differential diagnosis of esophageal eosinophilia. The finding of eosinophils on esophageal biopsy in isolation cannot be equated to a diagnosis of EoE. There are a number of gastrointestinal and systemic conditions that can cause eosinophils to infiltrate the esophageal mucosa. These include other eosinophilic gastrointestinal disorders such as eosinophilic gastroenteritis, Crohn's disease with esophageal involvement, pill esophagitis, connective tissue diseases, infections (fungal, viral, and parasitic), drug reactions, hypereosinophilic syn-

EoE Diagnostic Pitfalls drome, achalasia, graft-versus-host disease, and others [2, 4]. Most of these can be excluded with a thorough history, physical exam, and basic laboratory tests. However, the two conditions that EoE most frequently must be differentiated from are GERD and PPI-REE.

GERD is a common cause of upper gastrointestinal symptoms such as heartburn, dysphagia, and chest pain, and these can overlap with EoE symptoms. In case-control studies, there are no single symptoms or endoscopic findings that can differentiate EoE from GERD in isolation [13-15]. There is also substantial histologic overlap, with esophageal eosinophilia, basal zone hyperplasia, and spongiosis seen in both EoE and GERD [13, 14]. Even very high esophageal eosinophil counts cannot distinguish the two conditions. As shown in a retrospective review of esophageal biopsies at a GERD referral center [16], eosinophil counts for GERD and EoE patients were markedly elevated and overlapped substantially: the EoE group had 51-204 eos/HPF and the GERD group had 20168 eos/HPF. In addition to this clinical overlap, the relationship between GERD and EoE can be even more complicated. GERD and acid exposure can lead to esophageal eosinophilia, GERD and EoE can independently coexist, it is possible that EoE could lead to reflux (via altered motility or mucosal function caused by inflammation), and it is possible that reflux could lead to EoE (via increased allergen presentation due to injured mucosa) [17].

Complicating the issue further is PPI-REE, the term used to describe esophageal eosinophilia that responds to PPI therapy. This phenomenon was first reported in a case series of 3 pediatric patients who presented with dysphagia, food impaction, and vomiting, were found to have esophageal eosinophilia suspicious for EoE, and had resolution of symptoms and normalization of esophageal biopsies after treatment with PPI [18]. Since then, a number of prospective and retrospective studies in both children and adults have reported a PPI response rate between 33 and $74 \%$ in subjects with $\geq 15$ eos/HPF on esophageal biopsy [19-28]. Interestingly, the mechanism of this response might be an acid-independent anti-inflammatory effect of the PPIs themselves. Recent data suggest that PPIs can inhibit IL-13- and IL-4-induced eotaxin-3 secretion from esophageal epithelial cells in the absence of acid $[29,30]$.

To avoid clinical pitfalls in EoE diagnosis, it is important to fully consider the differential diagnosis of esophageal eosinophilia. GERD and PPI-REE will be the two most common alternatives to EoE when eosinophils are found on esophageal biopsy. As of yet, it is unclear wheth- 
er PPI-REE represents an independent condition, a subtype of GERD, a subphenotype of EoE, or potentially all three. However, because of the high rates of response to PPIs, EoE currently cannot be diagnosed without a formal PPI trial $[2,4]$.

\section{Endoscopic Pitfalls in EoE Diagnosis}

Endoscopic evaluation in patients with suspected EoE is used to evaluate for signs of EoE, for other alternative explanations for symptoms, and to obtain esophageal biopsies $[2,4]$. Endoscopic pitfalls related to EoE diagnosis include failing to recognize the findings of EoE and not obtaining adequate esophageal biopsy samples. The typical endoscopic findings of EoE have been well described and include esophageal rings, narrowing, or strictures; linear furrows; white plaques or exudates; decreased vasculature, pallor or edema, and mucosal fragility, also termed crêpe-paper mucosa [2, 3, 31-34]. However, in a small proportion of subjects (less than 5-10\%), the esophageal mucosa may appear normal, and the diagnosis will be missed if biopsies are not obtained $[35,36]$.

While the above endoscopic findings are common, they are not universal and do not fully differentiate EoE from other conditions such as GERD and PPI-REE. In a meta-analysis of 100 studies assessing more than 4,600 subjects with EoE and more than 2,700 non-EoE controls [36], the prevalence of individual EoE endoscopic findings varied substantially and were not universal. For example, esophageal rings were seen in $44 \%$, linear furrows in $48 \%$, white plaques in $27 \%$, and decreased vascularity in $41 \%$. However, rings and strictures were more common in adults, linear furrows and decreased vascularity were more common in children, and all findings were more common in prospective studies where at least one endoscopic abnormality was observed in $93 \%$ of subjects. This study also showed that the sensitivity and specificity of these findings were not sufficient for diagnosis of EoE alone. In addition to this, other studies have shown that there can be substantial inter- and intraobserver variability in recognition of endoscopic findings of EoE [37]. To address this variability, a new endoscopic classification system, termed the EoE Endoscopic Reference Score (EREFS), has recently been validated for identification of exudates, rings, edema, furrows, and strictures found in the esophagus of patients with EoE [38]. The hope is with use of this system and its accompanying atlas, recognition and reporting of endoscopic findings of EoE will become more standardized.
Because the endoscopic findings associated with EoE are not pathognomonic, it is crucial to obtain esophageal biopsies in all patients with suspected EoE regardless of the endoscopic appearance. However, there has been substantial variation in biopsy protocols both in the literature and in reported practice pattern surveys $[10,35]$. This is problematic because the eosinophilic infiltration in EoE can be patchy; therefore, if the esophagus is insufficiently sampled, the diagnosis can be missed. For example, levels of esophageal eosinophilia can vary widely not only throughout locations in the esophagus but also within biopsies themselves [39-41]. In two important papers, it has been clearly shown that increasing the number of biopsies increases the diagnostic sensitivity, which is maximized after at least 5 biopsies are taken $[41,42]$. The current guidelines recommend at least 2-4 biopsies from at least two different locations (i.e. proximal and distal) in the esophagus $[2,4]$.

To avoid endoscopic pitfalls in EoE diagnosis, it is important to understand that the 'classic' endoscopic findings of EoE are common, but not universal and not specific to EoE. In addition, use of a newly validated endoscopic classification system will help to standardize the reporting and reliability of endoscopic findings. However, regardless of the endoscopic appearance, esophageal biopsies should be obtained to assess for esophageal eosinophilia. The current recommendation is that at least 2-4 biopsies should be taken from both the proximal and distal esophagus to maximize the sensitivity for diagnosing EoE.

\section{Histologic Pitfalls in EoE Diagnosis}

After esophageal biopsies are obtained, thorough and accurate pathologic assessment is required. Pitfalls of histologic assessment include variability in diagnostic criteria and in eosinophil count quantification. Prior to the publication of the 2007 guidelines, there was substantial variability in diagnostic thresholds of esophageal eosinophilia reported throughout the literature, with 10 different values used (ranging from 5 to 30 eos/HPF), and with more than one third of the studies not reporting an EoE disease definition [35]. After adoption of a cut-point of 15 eos/HPF [3], there was less variability in the literature and in practice, though several cut-points (ranging from 15 to 30 eos/HPF) are still frequently used $[10,12]$. This variability is compounded because different microscopes have different HPF areas [35]. This means that for a constant eosinophil density (measured in eos $/ \mathrm{mm}^{2}$ rather 
Fig. 1. Clinical, endoscopic, and histologic pitfalls in diagnosing EoE. When EoE is suspected clinically and initial endoscopy shows at least 15 eos/HPF, EoE is still not yet diagnosed. The differential diagnosis of esophageal eosinophilia must be fully considered, and in particular GERD and EoE must be distinguished and PPI-REE must be excluded. After a PPI trial, a repeat endoscopy is required. Endoscopically, typical findings of EoE should be recognized, but these are not always specific for EoE. Additionally, adequate numbers of esophageal biopsies must be obtained from at least two esophageal locations regardless of the endoscopic appearance. Histologically, the peak eosinophil count should be determined, with the recognition that the absolute count might vary by pathologist based on the HPF size of a given microscope.

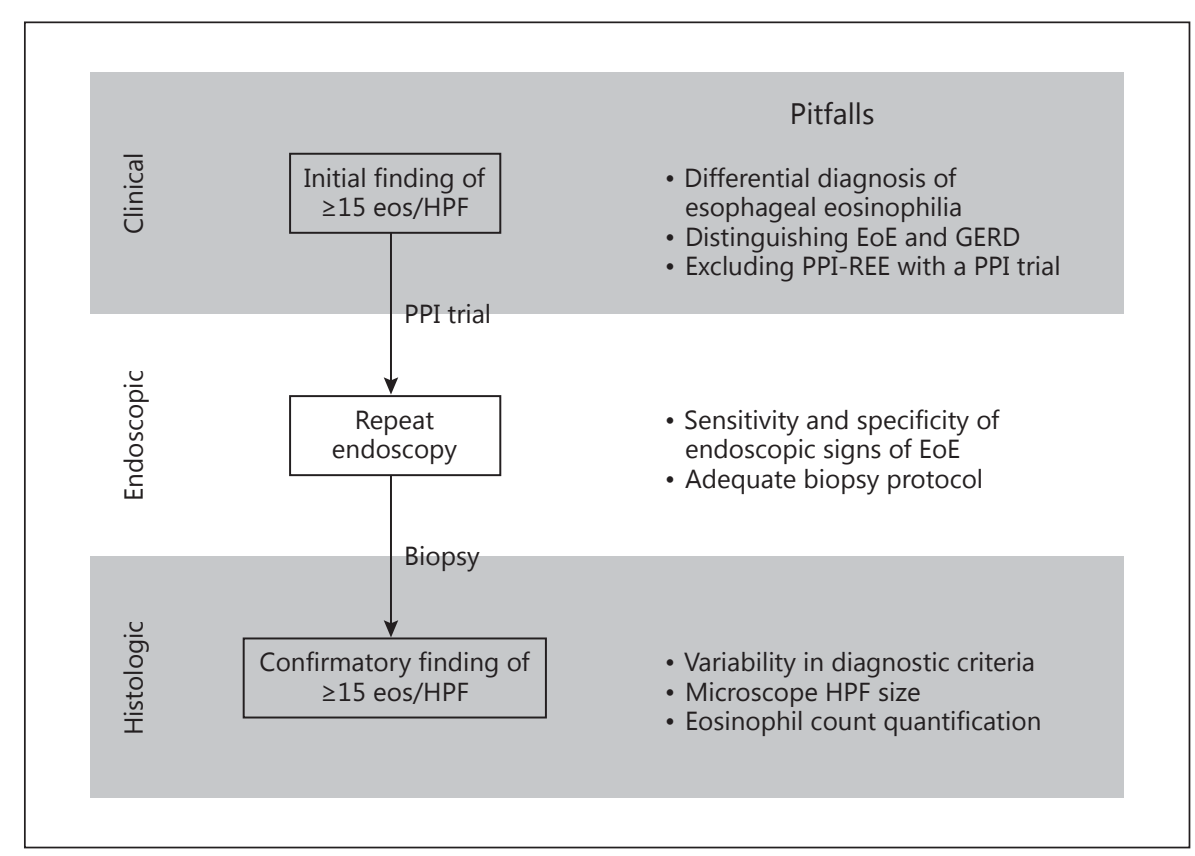

than eos/HPF), a microscope with a small field would yield a lower eosinophil count, while one with a larger field would find a higher count. Depending on the cutpoint used for diagnosis, a patient may or may not be histologically classified as EoE based solely on the microscope's field size, but instead on the underlying density of esophageal eosinophilia. For this reason, in the future it may be desirable to report eosinophil density $\left(\mathrm{eos} / \mathrm{mm}^{2}\right)$ rather than the eosinophil count (eos/HPF).

In addition to this variability by microscopy field, a number of other issues must be considered in order to determine the esophageal eosinophil count. For example, where in the biopsy specimen should the pathologist make the eosinophil count? Should the maximum number of eosinophils be quantified in any one HPF, should the total number in the entire specimen be counted, or should the mean value, recently termed the eosinophil load [43], be calculated from assessment of multiple HPFs throughout the sample? Typically, the peak number of eosinophils has been traditionally reported [2]. Additionally, a standardized approach for determining maximum eosinophil counts using digitized biopsy slides has been validated and has excellent inter- and intraobserver reliability between pathologists [44].

To avoid histologic pitfalls in the diagnosis of EoE, it is recommended that a diagnostic threshold of 15 eos/ HPF be used in clinical practice. However, it is important to be cognizant that the count will depend on the size of the microscope's HPF, so understanding how a collaborating pathologist quantifies eosinophils is key in order to interpret the results. Standard practice is to report the peak eosinophil count, but consideration could be given to following a validated eosinophil count quantification protocol.

\section{Conclusions}

The publication and updating of consensus guidelines for EoE has served to standardize the requirements for diagnosis of EoE. EoE is a clinicopathologic condition which is diagnosed when symptoms of esophageal dysfunction (such as dysphagia, food impaction, chest pain, or heartburn) are present in the setting of esophageal eosinophilia ( $\geq 15 \mathrm{eos} / \mathrm{HPF}$ ), but in the absence of competing causes of esophageal eosinophilia [2-4]. However, diagnosis of EoE is not always straightforward. A major reason for this is that there is no single clinical, endoscopic, or histologic sign that is pathognomonic for EoE, and the entire clinical picture must be considered before a diagnosis can be made. At each stage, pitfalls are encountered (fig. 1). Clinically, the differential diagnosis of esophageal eosinophilia must be fully considered, and in particular GERD and EoE must be distinguished and PPI-REE must be excluded. Endoscopically, typical findings of EoE should be recognized, but adequate numbers of esopha- 
geal biopsies must be obtained from at least two esophageal locations regardless of the endoscopic appearance, as tissue samples are currently required to make the diagnosis. Histologically, the peak eosinophil count should be determined, with the recognition that the absolute count might vary by pathologist based on the HPF size of a given microscope. Knowledge of these pitfalls allows the practitioner to avoid making errors in diagnosis, decrease diagnostic delay, and prescribe treatments for EoE to those who will most benefit from them. Given that the field is rapidly advancing, it is likely that novel techniques will change the paradigm for diagnosis of EoE in the future and minimize diagnostic pitfalls.

\section{Disclosure Statement}

Dr. Dellon receives research support from NIH, AGA, ACG, CURED Foundation, Meritage Pharma, and AstraZeneca. Dr. Dellon is a consultant for Novartis, Aptalis, and Receptos.

\section{References}

$\checkmark 1$ Katzka DA: Eosinophilic esophagitis: from $>12$ Sperry SL, Shaheen NJ, Dellon ES: Toward rookie of the year to household name. Clin Gastroenterol Hepatol 2009;7:370-371.

-2 Liacouras CA, Furuta GT, Hirano I, et al: Eosinophilic esophagitis: updated consensus recommendations for children and adults. J Allergy Clin Immunol 2011;128:3-20.e6.

3 Furuta GT, Liacouras CA, Collins MH, et al: Eosinophilic esophagitis in children and adults: a systematic review and consensus recommendations for diagnosis and treatment. Gastroenterology 2007;133:1342-1363.

4 Dellon ES, Gonsalves N, Hirano I, et al: ACG clinical guideline: evidence based approach to the diagnosis and management of esophageal eosinophilia and eosinophilic esophagitis (EoE). Am J Gastroenterol 2013;108:679-692.

5 Dellon ES: Diagnosis and management of eosinophilic esophagitis. Clin Gastroenterol Hepatol 2012;10:1066-1078.

6 Landres RT, Kuster GG, Strum WB: Eosinophilic esophagitis in a patient with vigorous achalasia. Gastroenterology 1978;74:12981301.

7 Attwood SE, Smyrk TC, Demeester TR, Jones JB: Esophageal eosinophilia with dysphagia. A distinct clinicopathologic syndrome. Dig Dis Sci 1993;38:109-116.

$\checkmark 8$ Straumann A, Spichtin HP, Bernoulli R, et al: Idiopathic eosinophilic esophagitis: a frequently overlooked disease with typical clinical aspects and discrete endoscopic findings (in German). Schweiz Med Wochenschr 1994;124:1419-1429.

9 Kelly KJ, Lazenby AJ, Rowe PC, et al: Eosinophilic esophagitis attributed to gastroesophageal reflux: improvement with an amino acidbased formula. Gastroenterology 1995;109: 1503-1512.

10 Peery AF, Shaheen NJ, Dellon ES: Practice patterns for the evaluation and treatment of eosinophilic oesophagitis. Aliment Pharmacol Ther 2010;32:1373-1382.

11 Spergel JM, Book WM, Mays E, et al: Variation in prevalence, diagnostic criteria, and initial management options for eosinophilic gastrointestinal diseases in the United States. J Pediatr Gastroenterol Nutr 2011;52:300-306. uniformity in the diagnosis of eosinophilic esophagitis (EoE): the effect of guidelines on variability of diagnostic criteria for EoE. Am J Gastroenterol 2011;106:824-832, quiz 833.

13 Dellon ES, Gibbs WB, Fritchie KJ, et al: Clinical, endoscopic, and histologic findings distinguish eosinophilic esophagitis from gastroesophageal reflux disease. Clin Gastroenterol Hepatol 2009;7:1305-1313.

14 Aceves SS, Newbury RO, Dohil R, et al: Distinguishing eosinophilic esophagitis in pediatric patients: clinical, endoscopic, and histologic features of an emerging disorder. J Clin Gastroenterol 2007:41:252-256.

15 Parfitt JR, Gregor JC, Suskin NG, et al: Eosinophilic esophagitis in adults: distinguishing features from gastroesophageal reflux disease: a study of 41 patients. Mod Pathol 2006; 19:90-96.

16 Rodrigo S, Abboud G, Oh D, et al: High intraepithelial eosinophil counts in esophageal squamous epithelium are not specific for eosinophilic esophagitis in adults. Am J Gastroenterol 2008; 103:435-442.

17 Spechler SJ, Genta RM, Souza RF: Thoughts on the complex relationship between gastroesophageal reflux disease and eosinophilic esophagitis. Am J Gastroenterol 2007;102: 1301-1306.

18 Ngo P, Furuta GT, Antonioli DA, Fox VL: Eosinophils in the esophagus - peptic or allergic eosinophilic esophagitis? Case series of three patients with esophageal eosinophilia. Am J Gastroenterol 2006;101:1666-1670.

19 Dranove JE, Horn DS, Davis MA, et al: Predictors of response to proton pump inhibitor therapy among children with significant esophageal eosinophilia. J Pediatr 2009;154:96-100.

20 Sayej WN, Patel R, Baker RD, et al: Treatment with high-dose proton pump inhibitors helps distinguish eosinophilic esophagitis from noneosinophilic esophagitis. J Pediatr Gastroenterol Nutr 2009;49:393-399.

21 Peterson KA, Thomas KL, Hilden K, et al: Comparison of esomeprazole to aerosolized, swallowed fluticasone for eosinophilic esophagitis. Dig Dis Sci 2010;55:1313-1319.
22 Molina-Infante J, Ferrando-Lamana L, Ripoll C, et al: Esophageal eosinophilic infiltration responds to proton pump inhibition in most adults. Clin Gastroenterol Hepatol 2011;9: 110-117.

23 Francis DL, Foxx-Orenstein A, Arora AS, et al: Results of ambulatory $\mathrm{pH}$ monitoring do not reliably predict response to therapy in patients with eosinophilic oesophagitis. Aliment Pharmacol Ther 2012;35:300-307.

24 Dellon ES, Speck O, Woodward K, et al: Prospective determination of the prevalence of PPI-responsive esophageal eosinophilia in patients with dysphagia undergoing upper endoscopy. Abstracts ACG Ann Sci Meet, Las Vegas, October 2012, Program No. 48.

25 Moawad FJ, Veerappan GR, Dias JA, et al: Randomized controlled trial comparing aerosolized swallowed fluticasone to esomeprazole for esophageal eosinophilia. Am J Gastroenterol 2013;108:366-372.

26 Schroeder S, Capocelli KE, Masterson JC, et al: Effect of proton pump inhibitor on esophageal eosinophilia. J Pediatr Gastroenterol Nutr 2013;56:166-172.

27 Mangla S, Singal G, Hornick JL, et al: Clinical predictors of response to proton pump inhibitors in patients with esophageal eosinophilia. Gastroenterology 2013;144(suppl 1):S495S496, Su1866.

28 Vazquez-Elizondo G, Ngamruengphong S, DeVault KR, Achem SR: The outcome of patients with esophageal eosinophilic infiltration after a PPI trial. Gastroenterology 2013; 144(suppl 1):S493, Su1857.

29 Cheng E, Zhang X, Huo X, et al: Omeprazole blocks eotaxin-3 expression by oesophageal squamous cells from patients with eosinophilic oesophagitis and GORD. Gut 2013;62: 824-832.

30 Zhang X, Cheng E, Huo X, et al: Omeprazole blocks STAT6 binding to the eotaxin-3 promoter in eosinophilic esophagitis cells. PLoS One 2012;7:e50037.

31 Straumann A, Rossi L, Simon HU, et al: Fragility of the esophageal mucosa: a pathognomonic endoscopic sign of primary eosinophilic esophagitis? Gastrointest Endosc 2003;57:407-412. 
32 Gupta SK, Fitzgerald JF, Chong SK, et al: Vertical lines in distal esophageal mucosa (VLEM): a true endoscopic manifestation of esophagitis in children? Gastrointest Endosc 1997;45:485-489.

33 Lim JR, Gupta SK, Croffie JM, et al: White specks in the esophageal mucosa: an endoscopic manifestation of non-reflux eosinophilic esophagitis in children. Gastrointest Endosc 2004; 59:835-838.

-34 Straumann A, Spichtin HP, Bucher KA, et al: Eosinophilic esophagitis: red on microscopy, white on endoscopy. Digestion 2004;70:109116.

35 Dellon ES, Aderoju A, Woosley JT, et al: Variability in diagnostic criteria for eosinophilic esophagitis: a systematic review. Am J Gastroenterol 2007;102:2300-2313.

36 Kim HP, Vance RB, Shaheen NJ, Dellon ES: The prevalence and diagnostic utility of endoscopic features of eosinophilic esophagitis: a meta-analysis. Clin Gastroenterol Hepatol 2012;10:988-996.e5.
37 Peery AF, Cao H, Dominik R, et al: Variable reliability of endoscopic findings with whitelight and narrow-band imaging for patients with suspected eosinophilic esophagitis. Clin Gastroenterol Hepatol 2011;9:475-480.

38 Hirano I, Moy N, Heckman MG, et al: Endoscopic assessment of the oesophageal features of eosinophilic oesophagitis: validation of a novel classification and grading system. Gut 2013;62:489-495.

39 Dellon ES, Speck O, Woodward K, et al: The patchy nature of esophageal eosinophilia in eosinophilic esophagitis: insights from pathology samples from a clinical trial. Gastroenterology 2012;142(suppl 2):Ab Su1129.

40 Saffari H, Peterson KA, Fang JC, et al: Patchy eosinophil distributions in an esophagectomy specimen from a patient with eosinophilic esophagitis: implications for endoscopic biopsy. J Allergy Clin Immunol 2012;130:798-800.
1 Gonsalves N, Policarpio-Nicolas M, Zhang Q, et al: Histopathologic variability and endoscopic correlates in adults with eosinophilic esophagitis. Gastrointest Endosc 2006;64: 313-319.

42 Shah A, Kagalwalla AF, Gonsalves N, et al: Histopathologic variability in children with eosinophilic esophagitis. Am J Gastroenterol 2009;104:716-721.

43 Straumann A, Conus S, Degen L, et al: Budesonide is effective in adolescent and adult patients with active eosinophilic esophagitis. Gastroenterology 2010;139:15261537.e1.

44 Dellon ES, Fritchie KJ, Rubinas TC, et al: Inter- and intraobserver reliability and validation of a new method for determination of eosinophil counts in patients with esophageal eosinophilia. Dig Dis Sci 2010;55:19401949. 